01,07

\title{
Изменения на поверхности гофрированной платиновой фольги под нагрузкой
}

\author{
() П.Н. Бутенко, В.Л. Гиляров, В.Е. Корсуков, А.В. Анкудинов, С.А. Князев, М.М. Корсукова, Б.А. Обидов \\ Физико-технический институт им. А.Ф. Иофрфе РАН, \\ Санкт-Петербург, Россия \\ E-mail: pavel.butenko@ioffe.mail.ru
}

Поступила в Редакцию 30 апреля 2021 г.

В окончательной редакции 30 апреля 2021 г.

Принята к публикации 7 мая 2021 г.

Исследованы изменения приповерхностных слоев гофрированных Pt-фольг, рассматриваемых в качестве мультимасштабных дифракционных решеток в процессе одноосного растяжения. Произведен анализ трансформации структуры и степени упорядоченности на разных этапах нагружения образцов, вплоть до их разрушения. С помощью концепции мультифрактального формализма предложен критерий эффективности использования данных фольг.

Ключевые слова: гофрированная фольга, дифракционная решетка.

DOI: 10.21883/FTT.2021.10.51390.102

\section{1. Введение}

В последние годы активно развивается область поверхностной инженерии (Surface Engineering). Основной задачей этого направления является изменение структуры поверхности твердого тела с целью придания ей определенных функциональных свойств [1-3]. С этой целью поверхность материала подвергается модификации (например: механическое, температурное, химическое, воздействие высокоэнергетическими частицами и пр.), изменяется ее структура, и как следствие, она приобретает требуемые для различных функциональных применений свойства. Вместе с тем, структура и свойства объема материала могут оставаться прежними. На сегодняшний день направление поверхностной инженерии находит применение в широком спектре приложений [4-6].

Ранее была получена модифицированная поверхность монокристаллической Pt-ленты, представляющая собой систему разномасштабных однонаправленных гофров, наложенных друг на друга [7,8]. Предложен механизм создания гофров посредствам формирования пространственно-модулированных дислокационных структур - полос локализованного скольжения с их выходом на поверхность кристалла [9]. Было выделено три масштаба, в которых расстояния между соседними гофрами, в которых составляет $70 \mathrm{~nm}, 1 \mu \mathrm{m}, 10 \mu \mathrm{m}$, что соответствует периодам решетки 1/14300, 1/1000, 1/100 mm [8,9]. Отмечалось, что такие структуры могут выполнять роль мультимасштабных дифракционных решеток. На каждом масштабе гофры параллельны друг другу и имеют пропорциональные параметры (соотношение высоты гофра к периоду дифракционной решетки). При использовании таких платиновых поверхностей,в качестве дифракционной решетки, последняя мо- жет работать в широком диапазоне излучения: от инфракрасного до мягкого рентгена [7-10]. Выяснилось, что такие решетки устойчивы к высоким температурам (до $1500 \mathrm{~K}$ ), предполагается, что они могут быть устойчивы к реакционным средам и к интенсивному излучению [8]. Учитывая большую интегральную площадь поверхности гофрированных Рt-структур, они могут применяться в качестве материалов, участвующих в процессах адсорбции и хемосорбции [11], а также использоваться в качестве подложек для укладки макромолекул (например, молекул ДНК и РНК), нанотрубок, т. е. в приложениях биосенсоров, эмиссионной электроники и пр. [12].

Вместе с тем, существует проблема устойчивости рельефа поверхности Pt гофрированных структур к внешним механическим воздействиям. Поскольку, толщина лент, на поверхности которых формируются гофры, относительно мала $(10-50 \mu \mathrm{m})$, а размеры самих гофров могут быть порядка $5 \mathrm{~nm}$, существует вероятность нарушения работы таких структур при деформировании гофров на различных масштабах вследствие воздействия внешних напряжений. Исходя из этого, необходимо изучение процессов, проходящих на поверхности в условиях механического нагружения.

Для исследования структуры поверхности материала, в том числе и при механических воздействиях, применяют различные экспериментальные методы зондовой микроскопии. Для анализа получаемых ими данных в последнее время интенсивно используются статистические методики, основанные на коллективном поведении неоднородностей рельефа поверхности, обладающих свойством самоподобия. Одним из таких методов является концепция мультифрактального формализма (MFF) [13]. Располагая геометрическими параметрами рельефа поверхности (полученными, например, атомносиловой микроскопией (AFM) или сканирующей тун- 
нельной микроскопией (STM)), применяя эту концепцию, можно описать текущее состояние поверхности и материала в целом и тенденции ее поведения при внешних воздействиях [14,15]. В предыдущих работах было отмечено, что структуры, сформированные в виде мультимасштабных Pt-решеток, можно рассматривать, как фрактальный объект, т. е. объект, обладающий самоподобием $[8,10]$. Известно, что основная характеристика таких структур - фрактальная размерность $\left(D_{f}\right)$. По этой величине можно судить о мере гладкости поверхности в целом или о мере иррегулярности. Однако поверхности реальных материалов неоднородны и анизотропны, следовательно, в общем случае должны учитываться детали локального скейлинга. В этом случае такой характеристикой является спектр сингулярностей, который может быть найденв результате применения концепции MFF.

Целью настоящей работы является комплексное исследование трансформации атомной структуры и геометрии рельефа поверхности, гофрированных Pt-фольг под воздействием одноосного механического растяжения. Для анализа изменений структуры верхних атомных слоев применена концепция мультифрактального формализма, с помощью которой определен критерий эффективности мультимасштабных дифракционных решеток.

\section{2. Материалы и методы}

Для получения Pt-ленты толщиной $10-50 \mu \mathrm{m}$, использовалась монокристаллическая платиновая проволока (чистота $99.99 \mathrm{wt} . \%$ ) диаметром $1 \mathrm{~mm}$, подвергшаяся холодной прокатке со степенью обжатия от 10 до 50. После этого поверхность ленты механически и электрохимически полировалась. Далее вырезались образцы с размерами $(30 \times 4) \mathrm{mm}$. Затем, вдоль бо́льших сторон образца (по обоим краям) были сделаны радиальные вырезы (по окружностям радиусом $10 \mathrm{~mm}$, расстояние в самом узком месте образца составило $2 \mathrm{~mm}$ ), которые будут являться концентраторами напряжений для локализации участка разрушения в последующих экспериментах по одноосному растяжению. На следующем этапе производилась рекристаллизация образцов ленты и очистка ее поверхности. Образцы устанавливались в специально сконструированное устройство для одноосного растяжения образцов, имеющее контакты для подвода электрического тока, пропускание через образец которого обеспечивало его нагрев. Очистка и рекристаллизация осуществлялись путем чередования циклов прогрева образцов в атмосфере кислорода при парциальном давлении кислорода $10^{-4}$ Ра при температуре $750-1100 \mathrm{~K}$ и отжига в сверхвысоком вакууме (UHV) при температуре 800-1900 К. Более подробно характеристики и последовательность циклов были описаны ранее [7-10]. Процесс производился в рабочем пространстве дифрактометра (комплекс Varian с камерой FC-12E). Температуру образца определяли с помощью пирометра. Эксперименты

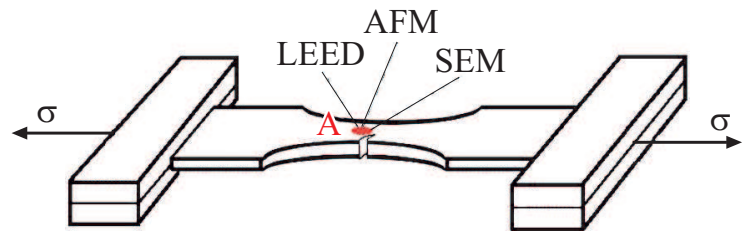

Рис. 1. Схема проведения экспериментов: показано положение области А, в которой непосредственно фиксировались результаты, относительно образовавшейся на конечном этапе нагружения магистральной трещины.

по одноосному растяжению проводились на следующем этапе, когда циклы рекристаллизации были завершены. Нагружение осуществлялось ступенчато, вплоть до $220 \mathrm{MPa}$, что является предразрывной нагрузкой, т.к. при следующем увеличении напряжения происходило разрушение образца.

В процессе температурных и механических воздействий на Pt-ленты, на разных этапах в камере дифрактометра производилось in situ изучение атомной структуры материала с помощью метода дифракции медленных электронов (LEED).

Геометрия поверхности образцов в свободном состоянии и под нагрузкой контролировалась методом сканирующей электронной микроскопии (SEM) на установке Seron Technology AIS2300C в режиме детектирования вторичных электронов. Основной задачей данного эксперимента являлось наблюдение за возникновением и развитием магистральной трещины на разных этапах нагружения непосредственно в области концентраторов напряжений, созданных радиальными вырезамии позиционирование участков, содержащих трещины.

Исследование рельефа поверхности ленты осуществляли с помощью метода AFM на установке Integra Aura в полуконтактном режиме. Изучали уже рекристаллизованные ранее в камере установки LEED, но не подвергавшиеся растяжению образцы. Для создания одноосного растягивающего напряжения (эксперимент in situ), также использовали устройство для растяжения образцов, которое устанавливалось в рабочее пространство AFM. Получаемые AFM-изображения имели латеральное разрешение $512 \times 512$ точек (на площадку сканирования), дополнительно были конвертированы в трехмерные численные массивы, которые использовались для статистического анализа.

На рис. 1 схематично изображено устройство для растяжения образцов, Pt-лента с вырезами, направления приложения растягивающей нагрузки (и образующаяся вследствие этого магистральная трещина), область А, в которой производились исследования.

Статистический анализ параметров геометрии поверхности Pt-лент включал в себя расчет значений фрактальной размерности и ряда параметров, получаемых методом MFF. Для определения $D_{f}$ использовался широко известный метод подсчета числа ячеек („Вох 

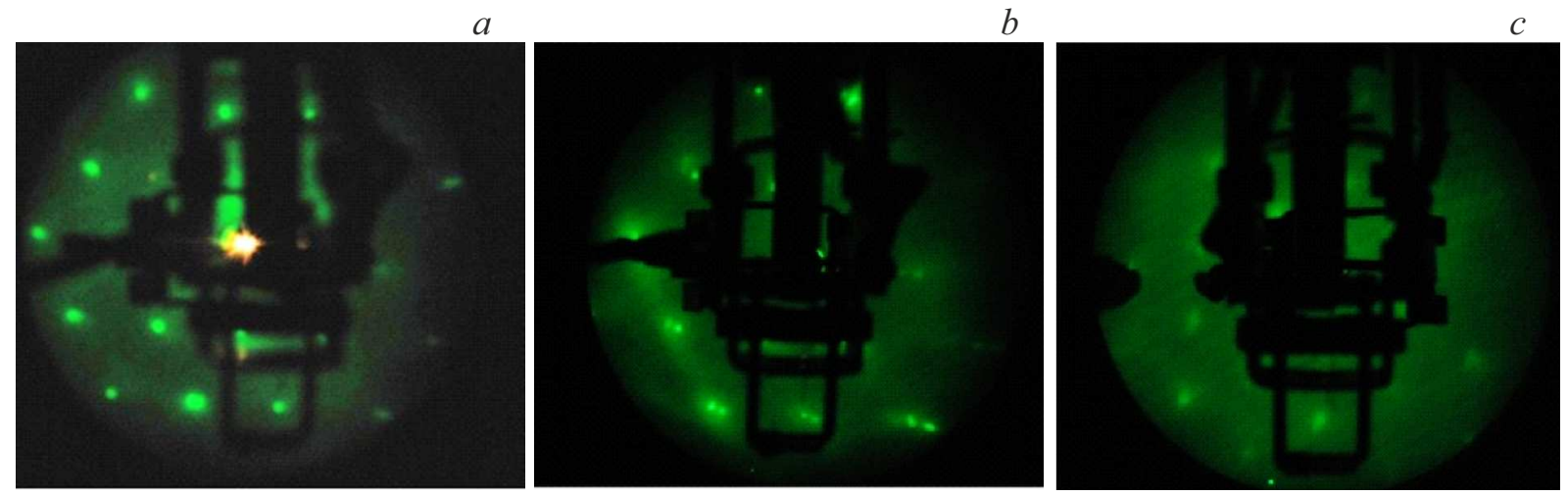

Pис. 2. LEED-картины, полученные от поверхности Рt-фольг, находящейся при разных значениях растягивающего напряжения: $a-$ исходный образец, $b-\sigma=25 \mathrm{MPa}, c-\sigma=220 \mathrm{MPa}$.

Counting“ method (BC)) [16]. Расчеты параметров MFF производились с помощью алгоритма реализованного в $[14,17]$. Приведем его основные этапы. Поверхность, полученная методом AFM, покрывается двумерной сеткой с ячейками размера $\varepsilon$. Положим, что $\varepsilon=l / L$, где $l-$ масштабируемый линейный параметр, $L-$ верхняя граница $l(L=512$, ограничена разрешением AFM-изображений), соблюдается условие: $l / L \leq 1 . P_{i, j}-$ вероятность распределения высот поверхностного профиля по ячейкам $i, j$, которая выражается как

$$
P(\varepsilon)_{i, j}=\frac{h_{i, j}}{\sum h_{i, j}}
$$

где $h_{i, j}-$ средняя высота ячейки размера $\varepsilon$, измеренная от глобального минимума профиля поверхности. В случае мультифрактальной поверхности для ячейки с индексами $i, j$ вероятность связана с размером: $P_{i, j} \propto \varepsilon^{\alpha}$, где $\alpha-$ это экспонента Липшица-Гельдера. Если множество - монофрактал, все ячейки имеют одинаковое значение $\alpha$, в противном случае - множество мультифрактал. При этом количество ячеек связано с их размером: $N_{\alpha=\operatorname{const}(\varepsilon)} \propto \varepsilon^{-f(\alpha)}$, где $f(\alpha)-$ спектр сингулярностей, являющийся непрерывной функцией от $\alpha$. Значение ординаты спектра $f(\alpha)$ определяет фрактальную размерность подмножества, имеющего определенное значение $\alpha$. Эти две характеристики позволяют численно оценить степень неоднородности распределения вероятностей. Спектр $f(\alpha)$ можно получить с помощью вычисления статистической суммы

$$
Z(q, \varepsilon)=\sum P_{i, j}^{q}(\varepsilon)=\varepsilon^{\tau(q)},
$$

где $q-$ порядок момента $(-\infty<q<\infty) ; \tau(q)-$ скейлинговая экспонента (или массовая экспонента), показывающая степень неоднородности мультифрактального множества, находится по наклону зависимости $Z(q, \varepsilon)$ от $\varepsilon$ в двойных логарифмических координатах (в области скейлинга она линейна). В свою очередь, если зависимость $\tau(q)$ нелинейна во всем диапазоне $q$, то такая поверхность является мультифракталом. Количественно $\alpha$ и $f(\alpha)$ связаны с $\tau(q)$ соотношениями Лежандра

$$
\left\{\begin{array}{l}
\alpha(q)=\frac{d \tau(q)}{d q} \\
f(\alpha)=q \alpha(q)-\tau(q) .
\end{array}\right.
$$

Из спектра сингулярностей, имеющего, как правило, куполообразную несимметричную форму, находят ширину спектра $\Gamma$ (разница максимального и минимального значения $\alpha$ ), которая и является некой обобщенной характеристикой поверхности, отражающей ее неоднородность.

\section{3. Результаты}

\section{1. Экспериментальное исследование атомной структуры лент $\mathbf{P t}$}

Дифракционные картины фольги $\mathrm{Pt}(111)$ в исходном состоянии (исходным материалом будем называть материал, прошедший циклы рекристаллизации, описание циклов см. выше) и нагруженном состояниях изображены на рис. 2. Видно, что в исходном состоянии (рис. $2, a$ ), LEED-картина имеет четкие точечные рефлексы, указывающие на то, что образцы имеют низкодефектную монокристаллическую структуру [18]. Нагрузка $25 \mathrm{MPa}$ приводит к раздвоению рефлексов (при этом они также четкие) (рис. $2, b$ ), что может быть вызвано несколькими факторами. Наиболее вероятные из них два: первый этап появления блоков, составляющих поликристалл и образование периодических одинаково ориентированных атомных ступенек, составляющих наноразмерные структуры типа $\operatorname{Pt}(9(111) \times 100)$ [19], т.е. дифракция на однонаправленных гофрированных наноструктурах [7]. Дальнейшее увеличение нагрузки вплоть до $220 \mathrm{MPa}$ и выдержка в течение $2 \mathrm{~h}$ влечет потускнение и размытие одинарных рефлексов (рис. 2,c). Это может быть объяснено, как разупорядочением структуры гофр, так и повышением блочности структуры (увеличение числа 


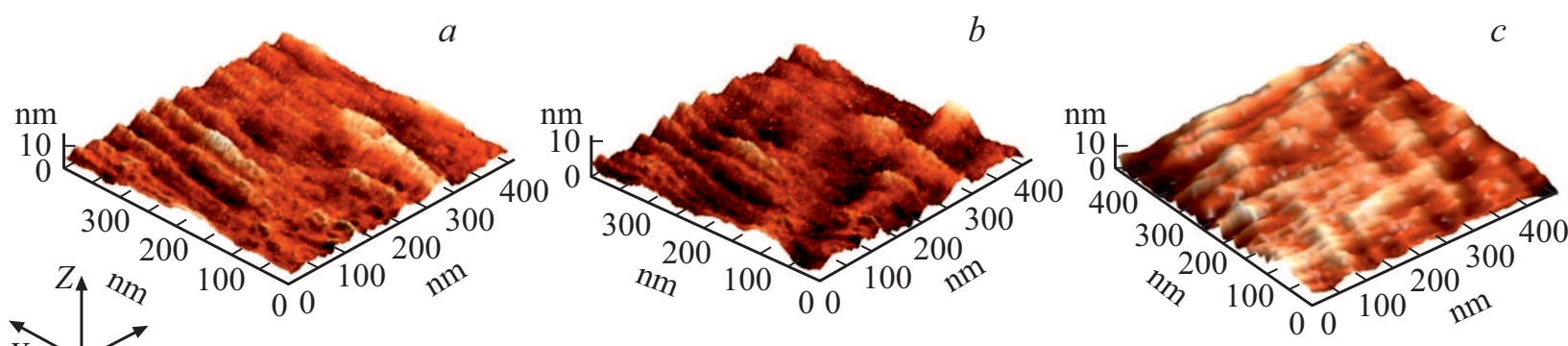

$d$

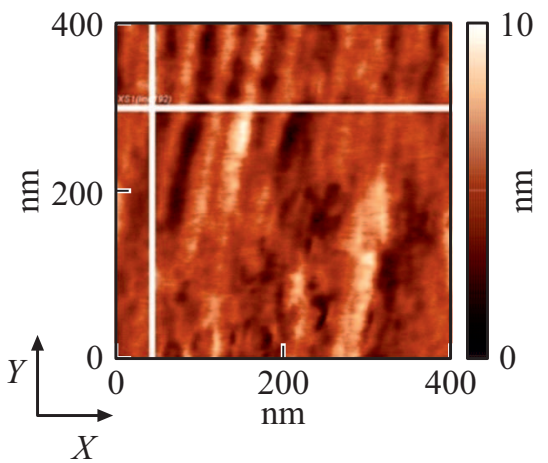

$e$
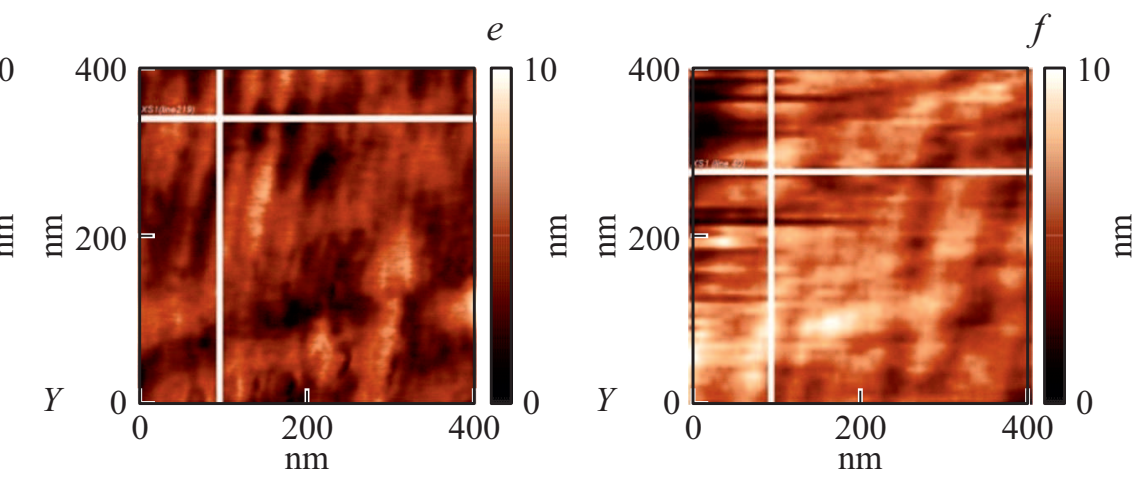

Рис. 3. Трехмерные и соответствующие им двумерные (с указанием выборочных сечений вдоль $O X$ и $O Y$ ) AFM-изображения поверхности $\mathrm{Pt}$ лент находящихся при разных значениях растягивающего напряжения: $a, d-$ исходный образец, $b, e-\sigma=25 \mathrm{MPa}$, $c, f-\sigma=220 \mathrm{MPa}$.
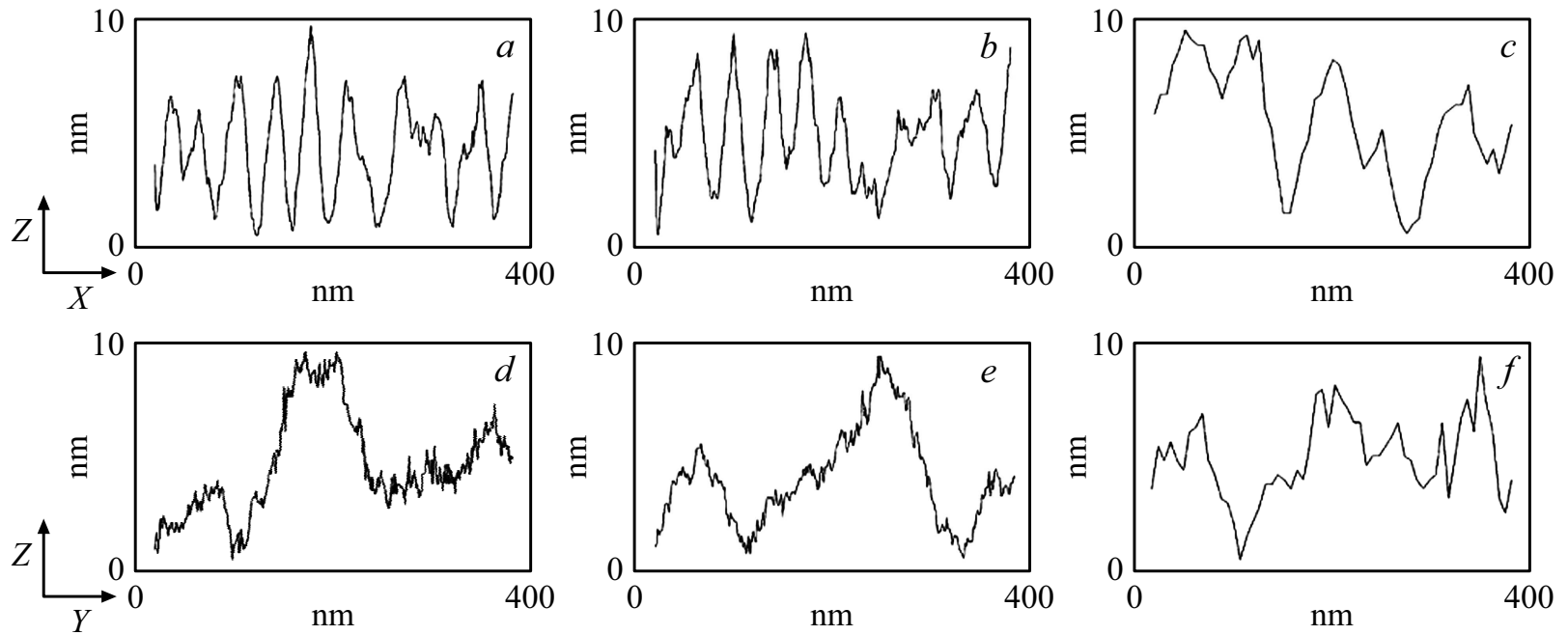

Рис. 4. Изображения выборочных сечений (построенных на рис. 3 ) в плоскости $X Z$ : $a-$ исходный образец, $b-\sigma=25 \mathrm{MPa}$, $c-\sigma=200 \mathrm{MPa} \mathrm{и} Y Z: d-$ исходный образец, $e-\sigma=25 \mathrm{MPa}, f-\sigma=220 \mathrm{MPa}$.

блоков, соответственно уменьшение их размеров) материала. Последнее может свидетельствовать о переходе материала в нанокристаллическое состояние, вплоть до его аморфизации [18].

\section{2. Экспериментальное исследование рельефа поверхности лент Pt}

Качественные изменения поверхностного рельефа образцов, подверженных вышеуказанным воздействиям, можно выявить, применив метод АFМ. Были сняты топо- граммы поверхностей исследуемых образцов в исходном состоянии и под нагрузкой на различных увеличениях, соответствующих площадкам: $1 \times 1 \mu \mathrm{m}, 3 \times 3 \mu \mathrm{m}$, $10 \times 10 \mu \mathrm{m}, 30 \times 30 \mu \mathrm{m}$. На рис. 3 показаны фрагменты $(400 \times 400 \mathrm{~nm})$ этих площадок в виде трехмерных и двумерных изображений. Гофрированная поверхность присутствует на рекристаллизованном исходном образце (рис. 3, $a$ и рис. $3, d$ ). На образце, подвергшемся растяжению $25 \mathrm{MPa}$, гофры выражены сильнее (рис. $3, b$ и $e$ ), регулярные параллельные структуры становятся более вытянутыми относительно $O Z$. 


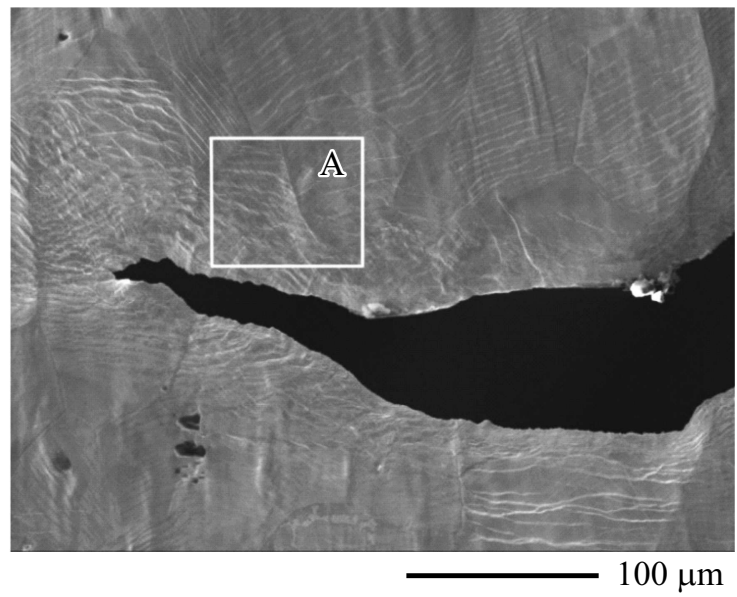

Рис. 5. SEM изображение магистральной трещины, распространяющейся в Pt-ленте при $\sigma=220 \mathrm{MPa}$ (предразрывное напряжение). Область А содержит участок, изображенный на рис. $3, c$.

Топограмма поверхности, находящихся при нагрузках близких к разрушению образца (220 МРa) в течение $2 \mathrm{~h}$, имеет сложный рельеф (рис. $3, c$ и рис. $3, f$ ), упорядоченных гофров на ней практически не наблюдается, тем не менее, просматривается система перпендикулярных друг другу плоских канавок. Более детально рельеф поверхностей можно проследить по сечениям, построенным перпендикулярно плоскости поверхности в двух направлениях (рис. 4). Сравнивая сечения, построенные перпендикулярно поверхности образцов в плоскости $Y Z$, образцов, находящихся в свободном состоянии и при нагрузке $25 \mathrm{MPa}$ (рис. $4, d$ и рис. $4, e$ соответственно), видно, что во втором случае регулярность профиля увеличивается. В случае высоких нагрузок профили, построенные вдоль $O X$ и $O Y$, становятся похожими, вместе с этим, оба имеют периодичность, но их качество (в смысле правильности формы элемента гофры) низкое (рис. $4, c$ и $f$ ).

Дополнительно геометрия поверхности образца визуализировалась с помощью метода SEM, который на бо́льших латеральных масштабах позволил наблюдать за развитием поверхностных дефектов в виде трещин. На рис. 5 изображена магистральная трещина, распространяющаяся от середины концентратора напряжений нормально к оси образца, совпадающей с направлением приложения нагрузки, которая составляла $220 \mathrm{MPa}$. Область А содержит участок, изображенный на рис. $3, c$, полученный в ходе AFM-эксперимента.

В итоге, качественное описание показывает, что геометрия поверхности на всех трех стадиях этого эксперимента, очевидно, связана с процессами структурных перестроек, проходящих на поверхности материала при увеличении нагрузки.

\section{3. Статистический анализ структуры поверхности лент Pt}

От качественного описания рельефа поверхности Pt-фольг, перейдем к количественному. Были выбраны площадки поверхностей Pt-фольг размерами $(1 \times 1) \mu \mathrm{m}$, $(3 \times 3) \mu \mathrm{m},(10 \times 10) \mu \mathrm{m},(30 \times 30) \mu \mathrm{m}$, которые находились в свободном состоянии и при разных значениях растягивающего напряжения (25-220 MPa). Рассчитанные для них значения фрактальных размерностей, отмечены на рис. $6, a$. На разных площадках при увеличении нагрузки значения $D_{f}$ имеют разнонаправленный характер.

Эти же участки в том же диапазоне нагрузок были проанализированыс помощью метода MFF. В результате для каждого участка были получены спектры сингулярностей и определены их ширины. На рис. $6, b$ приведены значения ширин спектров сингулярностей $(\Gamma)$ в зависимости от возрастания приложенной растягивающей нагрузки $(\sigma)$. Зависимость ведет себя немонотонно: после приложения напряжения наблюдается сужение спектра;
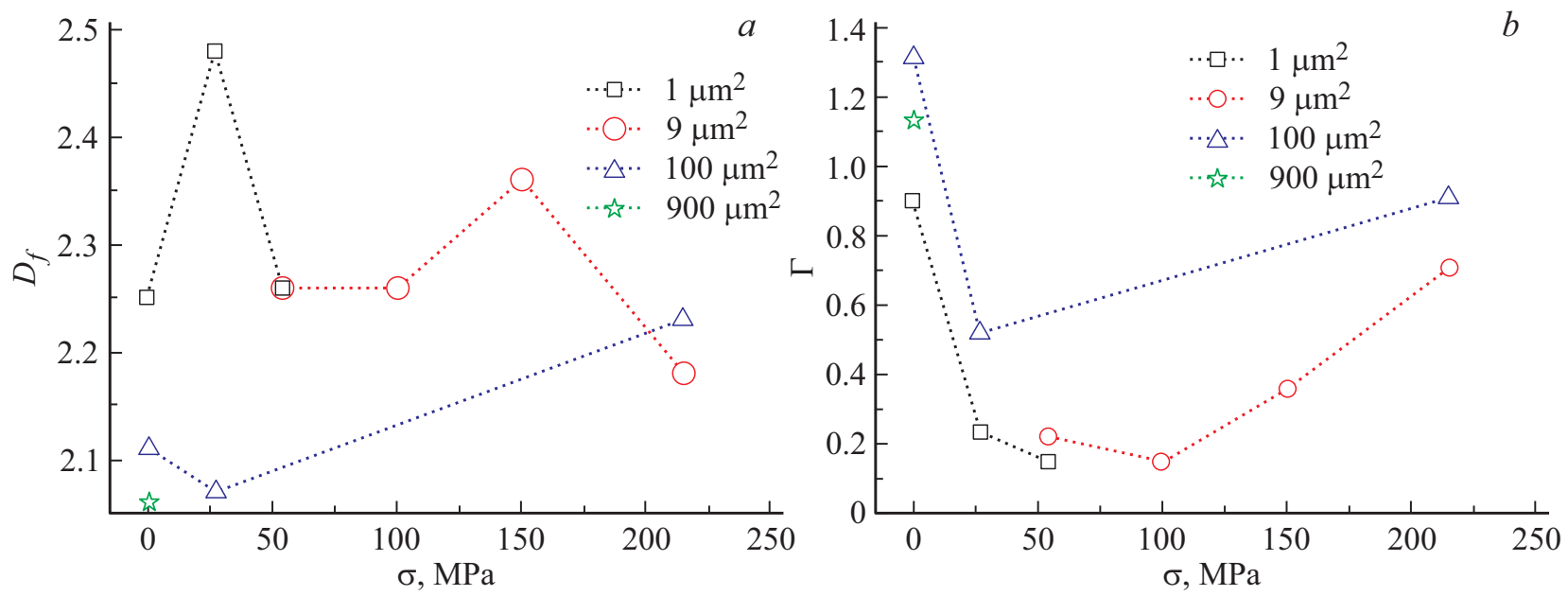

Рис. 6. Изменение значений фрактальных размерностей $(a)$ и ширин спектров сингулярностей $(b)$ поверхности Рt-лент при увеличении одноосного растягивающего напряжения, рассчитанные на различных масштабах. 
при последующем увеличении нагрузки (приближение к точке разрыва) ширина спектра сингулярностей увеличивается. Кроме того, значения ширин, вычисленные при одинаковых $\sigma$ для разных площадок,существенно отличаются.

\section{4. Обсуждение}

Интерпретируя полученные в работе результаты, необходимо учитывать, что методы LEED и AFM работают с разными слоями материала. LEED-картины могут отражать степень кристалличности приповерхностных слоев образца; при энергиях 50-100 eV эта глубина составляет 5-6 монослоев. В то же время, AFM-топограммы дают информацию о рельефе самой поверхности на микрометровом разрешении в латеральной плоскости и нанометровом в нормальном направлении к поверхности (не глубже поверхности внешнего монослоя).

Процесс приложения нагрузки в проведенном эксперименте фактически разделен на три стадии: исходное состояние; начальная нагрузка $(\sim 20 \%$ от разрывной нагрузки); нагрузка перед разрушением образца $(\sim 80 \%$ от разрывной нагрузки).

Анализ данных LEED-картины (рис. 2,a) образца в исходном состоянии показывает, что приповерхностные слои образца представляют собой структуру близкую к монокристаллической. AFM-изображения (рис. 3, $a, d$ ) демонстрируют сформированную гофрированную поверхность (регулярная система гофров с периодом порядка $30 \mathrm{~nm}$ ), подобную той, которая наблюдалась ранее [7-10]. Значения ширин спектров сингулярностей высокие (рис. 6, $b$ ), что указывает на неоднородность рельефа гофрированной поверхности и на то, что она является мультифракталом.

Возникающее раздвоение дифракционных рефлексов (рис. 2,b), после приложения нагрузки, очевидно, вызвано процессами потери монокристалличности приповерхностных слоев образца $[7,19]$. Рельеф поверхности достаточно гладкий: высоты неоднородностей примерно в 40 раз меньше их латеральных размеров (рис. 4), следовательно, он не должен вносить существенный вклад в изменение дифракционной картины. Вместе с тем, на этом этапе гофрированная структура поверхностного монослоя материала становится более упорядоченной, что отражают AFM-изображения (рис. $3, b, e$ ). В результате, структурирования поверхности повышается ее фрактальная размерность и уменьшается ширина спектра сингулярностей, т. е. поверхность демонстрирует тенденцию к монофрактализации. Последнее указывает на гомогенизацию поверхности в латеральной плоскости, однако это не противоречит факту потери монокристалличности, т. к. в данном случае рассматриваются процессы, протекающие параллельно, но на разных масштабах в нормальном направлении.
Наконец, на предразрывных нагрузках (непосредственно перед разрушением образца) LEED картины показывают размытие дифракционных рефлексов, (рис. 2,c), измельчение блоков и переход поликристалла в еще более разориентированное состояние, вплоть до аморфного, что отмечалось ранее [10]. На AFM-изображениях, полученных в области прохождения магистральной трещины, фиксируется укрупнение рельефа поверхности (рис. $3, c, f)$ и замена изначальной системы гофров на перпендикулярно расположенные канавки совместно с разупорядочением структуры. На данном этапе величина Г увеличивается, следовательно, сама поверхность становится вновь гетерогенной.

Ранее было отмечено, что в фольгах Pt при относительно малых нагрузках (20-80 MPa) трансформации структуры на атомном уровне (а именно, атомные ступеньки и наногофры) подвергается лишь малая часть образца, однако, с увеличением нагрузки эта часть увеличивается вместе разупорядочением структуры [18]. Согласующуюся с этим тенденцию имеет характеристика $D_{f}$ (рис. $\left.6, a\right)$. По мере увеличения нагрузки максимальные значения фрактальной размерности смещаются от малых к большим латеральным масштабам. Так, в образцах, находящихся в предразрывном состоянии $(\sigma=220 \mathrm{MPa})$, на самых крупных масштабах $\left(100 \mu \mathrm{m}^{2}\right)$, значения Df достигают максимума в то время, как на меньшем (предыдущем по уровню) увеличении $\left(9 \mu \mathrm{m}^{2}\right)$ значение $D_{f}$ уже успело понизиться по сравнению с предыдущей нагрузкой $(150 \mathrm{MPa})$. В свою очередь на еще меньшем увеличении $\left(1 \mu \mathrm{m}^{2}\right)$, максимум значение $D_{f}$ испытывало при начальной нагрузке $(\sigma=25 \mathrm{MPa})$. Таким образом, при приложении растягивающего напряжения, очевидно, что структурирование поверхности начинается на малых латеральных масштабах и по мере увеличения нагрузки, затрагивает все бо́льшие.

Также следует отметить, что различия в значениях ширин спектров сингулярностей, полученные при одинаковых нагрузках, но на площадках различных размеров объясняются тем, что при расширении области сканирования, каждый раз добавляются площади, имеющие собственный рельеф, что приводит к уширению спектров. Действительно, на рис. $6, b$ во всех четырех случаях при одинаковых нагрузках видно однозначное увеличение Г с ростом размера площадки.

\section{5. Заключение}

Процесс трансформации модифицированной поверхности монокристаллических Pt-лент под действием механического растяжения имеет следующую последовательность:

- Изначально приповерхностный слой платиновой фольги на глубине в несколько атомных слоев монокристалличен (регулярен). В то же время, самый верхний монослой нерегулярен, что подтверждается высокими значениями ширин спектров сингулярностей. Однако эта 
нерегулярность имеет столь малый масштаб по нормали к поверхности, что она не может оказать сколь-нибудь значительное влияние на дифракционную картину LEED.

- При приложении нагрузки начинается структурирование верхнего монослоя, распространяющееся с малых латеральных масштабов на все бо́льшие при повышении нагрузки (увеличение фрактальной размерности совместно с уменьшением ширины спектра сингулярностей). На бо́льшей глубине начинается процесс потери монокристалличности - процесс разупорядочивания. На данном этапе ожидается, что дифракционные решетки могут обладать максимальной эффективностью.

- При предразрывных нагрузках на поверхности наблюдается магистральная трещина. Это приводит к релаксации напряжений в ее окрестности и новому разупорядочению поверхностного монослоя. Спектр сингулярностей поверхности при этом расширяется, хотя гофры на данном этапе практически отсутствуют. Увеличение ширины мультифрактального спектра в данном случае может служить показателем понижения функциональности поверхности. На бо́льшей глубине продолжается разупорядочение структуры на нанометровом нормальном масштабе, вплоть до ее аморфизации.

Исходя из проведенного исследования, ожидается, что наибольшую эффективность работы дифракционные гофрированные решетки, созданные на основе Pt-лент, способны проявлять при нахождении под нагрузкой порядка $10 \%$ от разрывной.

\section{Благодарности}

Авторы выражают благодарность В.Н. Вербицкому за помощь в экспериментах, проведенных с помощью сканирующей электронной микроскопии.

\section{Конфликт интересов}

Авторы заявляют, что у них нет конфликта интересов.

\section{Список литературы}

[1] M. Shen, S. Zhu, F. Wang. Nature Commun. 7, 13797 (2016).

[2] M. Mozetic. Materials 12, 441 (2019).

[3] K. Prajwal, G.L. Priyanka, M.A. Hasan, A.C.M. Esther, N. Sridhara, A. Rajendra, S.B. Arya, A. Dey. Surf. Eng. 37, 3, 400 (2021).

[4] V. Shibe, V. Chawla. IJRMET 3, 2, 85 (2013).

[5] A. Hadipour, M. Rahsepar, H. Hayatdavoudi. Surf. Eng. 35, 10,883 (2019).

[6] T. Hanawa. Dental Mater. J. 39, 1, 24 (2020).

[7] В.Е. Корсуков, С.А. Князев, А.Л. Буйнов, М.М. Корсукова, С.А. Немов, Б.А. Обидов. ПЖТФ 39, 8, 55 (2013).

[8] В.Е. Корсуков, А.В. Анкудинов, П.Н. Бутенко, С.А. Князев, М.М. Корсукова, Б.А. Обидов, И.П. Щербаков. ПЖТФ 40, 18, 1 (2014).

[9] В.Е. Корсуков, Г.А. Малыгин, М.М. Корсукова, И.А. Няпшаев, Б.А. Обидов. ФТТ 57, 12, 2463 (2015).
[10] V.E. Korsukov, P.N. Butenko, A.G. Kadomtsev, M.M. Korsukova, V.S. Levitskii, I.A. Nyapshaev, B.A. Obidov. Nanosystems: phys., chem., mathem. 9, 1, 58 (2018).

[11] S. Vajda, M.J. Pellin, J.P. Greeley, Ch.L. Marshall, L.A. Curtiss, G.A. Ballentine, J.W. Elam, S. Catillon-Mucherie, P.C. Redfern, F. Mahmood, P. Zapol. Nature Mater. Lett. 8, 213 (2009).

[12] E.O. Popov, A.A. Pashkevich, A.O. Pozdnyakov, O.F. Pozdnyakov. J. Vac. Sci. Technol. B 26, 745 (2008).

[13] T.Tél. Z. Naturforsch 43, 12, 1154 (1988).

[14] M. Nasehnejad, M. Cholipour Shahraki, G. Nabiyouni. Appl. Surf. Sci. 389, 735 (2016).

[15] П.Н. Бутенко, В.Л. Гиляров, В.Е. Корсуков, М.М. Корсукова, Б.А. Обидов. ЖТФ 90, 2, 217 (2020).

[16] G. Gonzato. Comp. Geosci. 24, 1, 95 (1998).

[17] Z.W. Chen, J.K.L. Lai, C.H. Shek. Phys. Lett. A 345, 218 (2005).

[18] С.А. Князев, В.Е. Корсуков. ФТТ 47, 5, 876 (2005).

[19] B. Lang, R.W. Joyner, G.A. Somorjai. Surf. Sci. 30, 2, 440 (1972).

Редактор К.В. Емцев 